\title{
O DESAFIO PARA O CONTROLE DOMICILIAR EM CRIANÇAS E ADOLESCENTES DIABÉTICAS TIPO 1
}

\author{
Maria Lúcia Zanetti ${ }^{1}$ \\ Isabel Amélia Costa Mendes² \\ Kátia Prado Ribeiro ${ }^{3}$
}

Zanetti ML, Mendes IAC, Ribeiro KP. O desafio para o controle domiciliar em crianças e adolescentes diabéticas tipo 1. Rev Latino-am Enfermagem 2001 julho; 9(4):32-6.

Estudo descritivo com mães de crianças e adolescentes portadores de diabetes tipo 1, com o objetivo de identificar os recursos disponiveis e de analisar as dificuldades que essas mães encontram para o controle domiciliar. Para o alcance dos objetivos, empregou-se entrevista dirigida, cujos resultados permitiram concluir que os instrumentos e equipamentos utilizados no domicílio para o controle do diabetes são escassos e, quando disponíveis, não são manuseados adequadamente. A comunicação das mães com a equipe de saúde mostrou-se limitada. Os resultados apontam para a necessidade de eqüidade no atendimento às famílias de crianças e adolescentes diabéticos tipo 1 , fornecendo apoio para que estas possam lidar com a criança portadora de doença crônica.

PALAVRAS CHAVE: criança, adolescente, diabetes mellitus tipo 1

\section{THE CHALLENGE OF CARING FOR CHILDREN AND ADOLESCENTS WITH TYPE-1 DIABETES AT HOME}

This is a descriptive study concerning mothers of children and adolescents with type-1 diabetes. Its purpose is to identify available resources and analyze the difficulties that these mothers find to control the disease at home. To that end, direct interviews were used and the results enabled us to reach the following conclusions: the resources used for the control of diabetes at home are scarce and, when available, they are not handled adequately; communication between mothers and the health care team was limited. The results also pointed to the need for equity in the assistance to families of children and adolescents with type-1 diabetes by providing support for such families to manage children with chronic diseases.

KEY WORDS: child, adolescent, type-1 diabetes

\section{EL DESAFÍO PARA EL CONTROL DOMICILIARIO EN NIÑOS Y ADOLESCENTES DIABÉTICOS TIPO 1}

Estudio descriptivo con madres de niños y adolescentes portadores de Diabetes tipo 1, con el objetivo de identificar los recursos disponibles y analizar las dificultades que esas madres encuentran para el control domiciliario. Para alcanzar los objetivos, realizamos una entrevista dirigida, cuyos resultados nos permitieron concluir: los recursos utilizados en el domicilio para el control de la diabetes son escasos y cuando están disponibles, no son utilizados adecuadamente. La comunicación de las madres con el equipo de salud se mostró limitada. Percibimos también la necesidad de equidad en la atención a las familias de niños y adolescentes diabéticos tipo 1, ofreciendo apoyo y soporte para que estos puedan lidiar con el niño portador de enfermedad crónica.

PALABRAS CLAVES: Niño, Adolescente, diabetes tipo 1

${ }^{1}$ Professor Doutor, e-mail: zanetti@eerp.usp.br; ${ }^{2}$ Professor Titular, Escola de Enfermagem de Ribeirão Preto da Universidade de São Paulo, Centro Colaborador da OMS para o desenvolvimento da pesquisa em enfermagem; ${ }^{3}$ Enfermeira, bolsista de Apoio Técnico CNPq 


\section{INTRODUÇÃO}

As recomendações para o controle domiciliar do diabético incluem auto-monitorização: da glicemia capilar, de múltiplas doses de insulina, das alterações nos padrões dietéticos a partir de reeducação alimentar e da realização de atividades físicas programadas, a fim de manter os níveis glicêmicos. Estas recomendações implicarão em mudanças de comportamento dos pacientes, profissionais de saúde e familiares ${ }^{(1)}$.

Na prática da clínica diária fatores como idade, crenças, valores, motivação pessoal, condições financeiras e doenças associadas, entre outros aspectos, podem influenciar na decisão do paciente ou do familiar em optar por um controle mais rígido, para o qual o profissional de saúde deve estar capacitado para orientá-lo e apoiá-lo ${ }^{(2)}$.

Assim, capacitar e dar suporte à pessoa diabética para 0 autocontrole domiciliar é uma responsabilidade conferida aos profissionais de saúde. Ocorre que na maior parte dos serviços de atendimento ao diabético, no Brasil, esses profissionais nem sempre encontram-se adequadamente preparados ou disponiveis ${ }^{(3)}$.

Nos últimos anos, muitos questionamentos têm sido feitos a respeito das dificuldades na implementação dos rígidos controles preconizados pelas recomendações do "Diabetes Control and Complication Trial Research Group" (DCCT) e também sobre suas implicações na prática clínica, nos programas educacionais, no autocontrole, na qualidade de vida e no custo do tratamento, especialmente nos serviços que não dispõem de recursos e de profissionais capacitados. Sendo assim, é necessário o desenvolvimento de políticas gerais visando a implantação de um programa efetivo de autocontrole do diabetes, a disponibilização de centros regionais para o controle da doença, número suficiente de médicos e especialistas, além de núcleos destinados a pesquisas, com o fim de identificar fatores que interfiram na decisão dos pacientes e de seus familiares quanto ao tratamento em questão, favorecendo a adesão ao autocontrole ${ }^{(3)}$.

Atentas a estas questões, interessamo-nos pelo estudo da problemática que envolve os recursos disponíveis e as dificuldades que as mães de crianças e adolescentes portadores de diabetes tipo 1 apresentam para a adesão ao controle domiciliar.

\section{OBJETIVO}

Identificar os recursos disponíveis e analisar as dificuldades que as mães de crianças e adolescentes diabéticos tipo 1 apresentam para o controle domiciliar.

\section{MATERIAL E MÉTODOS}

Trata-se de um estudo descritivo e seu desenho atende aos pré-requisitos de um estudo de caso. A população foi constituída por 30 (trinta) mães de crianças e adolescentes diabéticos tipo 1 , matriculados em escolas públicas e particulares de $1^{\circ}$ e $2^{\circ}$ graus de Ribeirão Preto-SP.

Para a coleta de dados utilizou-se um roteiro de entrevista semi-estruturada, considerando as variáveis relacionadas ao controle domiciliar, contendo duas partes e 12 questões semi-abertas. Selecionou-se esta população através da aplicação de um formulário nas escolas para identificação de crianças e adolescentes portadores de diabetes. Posteriormente, os pesquisadores dirigiram-se às residências das famílias esclarecendo às mães o objetivo e a natureza da investigação, obtendo delas o consentimento formal para a coleta de dados e assegurando-lhes o anonimato e o sigilo das respostas.

Estes mesmos pesquisadores realizaram a coleta de dados junto às mães, nos meses de agosto e setembro de 1998, na residência da família, em ambiente privado para que não houvesse interferência garantindo, desse modo, tranqülidade na emissão das respostas. As entrevistas tiveram duração média de sessenta minutos e as respostas às questões semi-abertas foram registradas no próprio instrumento, concomitantemente à entrevista.

$\mathrm{Na}$ análise dos dados foram atribuídos códigos específicos às variáveis relacionadas ao controle domiciliar, procedendo-se à transcrição das questões codificadas em uma planilha. A estrutura do banco de dados foi confeccionada a partir das planilhas, sendo 0 mesmo formatado no programa FOX PRO-2. A análise obedeceu às normas preconizadas para um estudo descritivo.

\section{RESULTADOS E DISCUSSÃO}

A apresentação e discussão dos resultados seguirá a linha dos recursos disponíveis e análise das dificuldades apresentadas pelas mães das crianças e adolescentes com diabetes mellitus tipo 1 , segundo as variáveis relacionadas ao controle do diabetes.

Ao entrevistarmos as $30(100 \%)$ mães sobre as fontes dos recursos disponíveis para aquisição da insulina a ser utilizada pelas crianças e adolescentes com diabetes mellitus tipo 1 , obtivemos que $17(56,7 \%)$ adquirem a insulina através de recursos próprios e 13 $(43,3 \%)$ em instituições governamentais. Dentre aquelas que obtêm o produto através de recursos governamentais, foram apontadas algumas dificuldades na aquisição dos frascos de insulina, pois, devido ao grande número de marcas existentes no mercado, nem sempre aquela prescrita pelo médico é a que está disponível nas instituições governamentais. Neste caso, a mãe tem sempre que retornar ao 
médico para trocar a receita médica. Acreditamos que o treinamento dos funcionários responsáveis pela distribuição de insulina muito contribuirá para minorar esta situação, seja em relação à espécie de origem, grau de purificação, tempo de ação e a concentração da mesma, independentemente das marcas existentes no mercado.

As mães que adquirem-na através de recursos próprios sabem que poderiam obtê-la gratuitamente em instituições governamentais; porém uma delas afirmou que não a adquire nestes serviços porque tem "...medo que a insulina não seja de boa qualidade e que a coisa pública não é boa..."

Particularmente, em relação à insulina, um órgão governamental do Ministério da Saúde que atende às necessidades do mercado nacional responde pelo controle de qualidade da insulina produzida no Brasil. Na Etiópia, por exemplo, onde o diabetes afeta um em cem dos seus habitantes e onde $40 \%$ dos diabéticos dependem de insulina para sobreviver, não há garantia de suprimento das necessidades de todos portadores de diabetes, pois o país encontra-se ainda subordinado à importação de insulina ${ }^{(4)}$.

Segundo a Organização Mundial da Saúde, muitas mortes, em vários países sub-desenvolvidos, ocorrem pela falta da insulina, pela disponibilidade limitada ou pelo alto custo da mesma. No entanto, para a população em estudo, a aquisição de insulina não constitui um determinante de interferência importante para o tratamento $e$ controle.

Quando indagamos sobre quem assumiu a responsabilidade pelo acompanhamento do filho, 27 (90,0\%) mães de crianças e adolescentes com diabetes tipo 1 referiram que elas próprias eram responsáveis; $2(6,7 \%)$ o pai e $1(3,3 \%)$ o pai e a mãe.

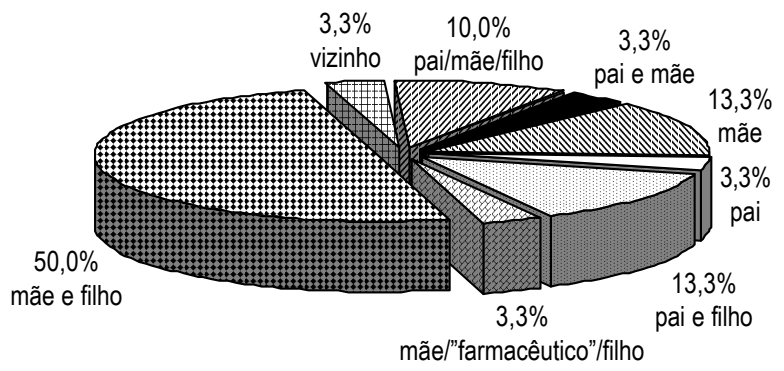

Figura 1 - Distribuição das mães de crianças e adolescentes com diabetes mellitus tipo 1, matriculados em escolas de $1^{\circ}$ e $2^{\circ} \mathrm{grau}$, segundo responsável pela administração de insulina, Ribeirão PretoSP, 1998.

Ao investigarmos quem assume a responsabilidade pela administração de insulina, identificamos que a aplicação em 15 $(50,0 \%)$ casos é efetuada pela mãe e filho; em $4(13,3 \%)$ pelo pai el ou filho e em outros $4(13,3 \%)$ apenas pela mãe.

Os resultados encontrados nesta investigação reforçam os $\operatorname{achados}^{(5)}$ em relação à atuação das mães frente à doença crônica dos filhos. Eles mostram que a responsabilidade das mães na administração de insulina é maior devido à sua participação no cuidado diário dos filhos, pois são as mães que os levam à consulta médica; que, freqüentemente, fazem as anotações referentes à dieta, aos testes de urina, a episódios de hipoglicemia, além de os acompanharem aos grupos de educação em diabetes. Partilhamos também da compreensão de que os problemas com os filhos são mais sentidos pelas mães do que pelos pais, as quais vivenciam mais a doença e os seus efeitos sobre os outros membros da família.

Estes dados indicam a necessidade da inclusão dos pais nos programas de educação em diabetes para crianças e adolescentes, programas que devem dispensar também às mães atenção especial, pois elas precisam de orientação e suporte para o cuidado diário do filho diabético, principalmente quanto à administração de insulina: função que deverá ser desempenhada pelo enfermeiro através de visitas domiciliares. Somente o enfermeiro terá condições de desenvolver objetivos realistas, de acordo com a idade e o nível de compreensão da criança e necessidades da familila $^{(6)}$.

Os dados mostram-nos que a maioria das mães aprendeu a administrar a insulina com profissionais da saúde, merecendo relevância o trabalho desenvolvido pelos auxiliares de enfermagem no processo educativo com $7(23,3 \%)$ delas e pelos "farmacêuticos" * com $6(20 \%)$ mães.

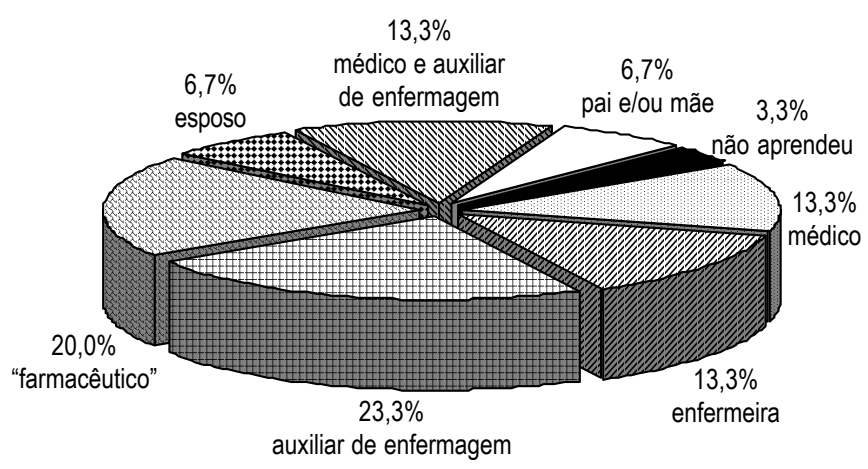

Figura 2 - Distribuição das mães de crianças e adolescentes com diabetes mellitus tipo 1 , matriculados em escolas de $1^{\circ}$ e $2^{\circ} \mathrm{grau}$, segundo aprendizado para administração de insulina, Ribeirão PretoSP, 1998.

Ao analisarmos os recursos humanos destinados aos ambulatórios especializados, necessários para 0 atendimento de diabéticos tipo 1, crianças ou adultos, presentes na proposta elaborada pela Secretaria Municipal de Saúde de Ribeirão Preto$\mathrm{SP}^{(7-8)}$ vimos que a composição da equipe multiprofissional inclui médicos, enfermeiros, nutricionistas, assistentes sociais, psicólogos

* Esta denominação foi apresentada pelas mães, independentemente da educação formal destes profissionais 
e educadores em saúde, sendo que os dois primeiros compõem a equipe mínima para 0 atendimento desta clientela.

Dentro da equipe multiprofissional, o enfermeiro é responsável pelo desenvolvimento de programas de treinamento relativos ao cuidado domiciliar da pessoa diabética, incluída a técnica de auto-aplicação de insulina. Porém, dados a este respeito são preocupantes, pois constatamos que somente $4(13,3 \%)$ mães aprenderam a auto-aplicação de insulina com este profissional, tendo tido maior desempenho os auxiliares de enfermagem e o "farmacêutico". Parece-nos que o enfermeiro não tem assumido este seu papel dentro da equipe multidisciplinar.

Em relação aos instrumentos e equipamentos utilizados na residência para o controle do diabetes, observamos que 22 (73,3\%) mães utilizam algodão, álcool, seringa, agulha; 5 (16,7\%) algodão, álcool, seringa, agulha e fitas reagentes; 2 (6,7\%) algodão, álcool, seringa, agulha e glucometer* e 1 (3,3\%) algodão, álcool, seringa, agulha e vitaje ${ }^{\star \star}$, o que demonstra não haver boa condição em termos de recursos materiais, para o desenvolvimento da terapêutica no domicílio.

Estes dados apontam ainda que a maioria das mães (73,3\%) utiliza apenas seringa, agulha, algodão e álcool e que 16,7\% possuem fitas reagentes na residência, mas não estão realizando 0 controle de urina e de sangue através das mesmas. As mães que possuem o glucometer $(6,7 \%)$ não sabem manuseá-lo e as que adquiriram o vitajet (3,3\%) não se adaptaram ao aparelho.

Diante de tais informações, reconhecemos que para atingir um bom controle metabólico do diabetes na criança e no adolescente é necessário melhorar o provimento de materiais e, sobretudo envolver efetivamente a família neste tratamento. Este envolvimento impõe-se por ser a família importante fator na redução de complicações para a criança, propiciando condições para uma vida mais saudável. Mas, para que os familiares participem do tratamento necessitam aprender a manusear os instrumentos e praticar o controle domiciliar diário do diabetes. Tal controle hoje é facilitado, pois existe no mercado nacional uma variedade de produtos e equipamentos, tais como: fitas reagentes para determinação de glicose no sangue, pesquisa de glicose e cetona na urina, aparelhos para auto-aplicação de insulina, entre outros.

Entretanto, as questões aqui levantadas sobre o controle domiciliar têm limitações por evidenciarem lacunas na terapêutica domiciliar. Diante disto, sentimos a necessidade de revisão do tratamento domiciliar, tendo em vista o controle metabólico. E, como sabemos, parte desta responsabilidade cabe aos enfermeiros.

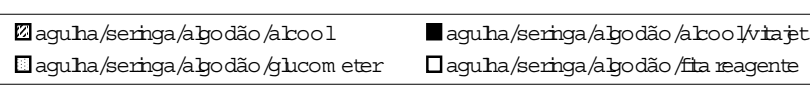

图aguha/seringa/abodão/glucom eter 口aguha/seringa/agodão/flia reagente

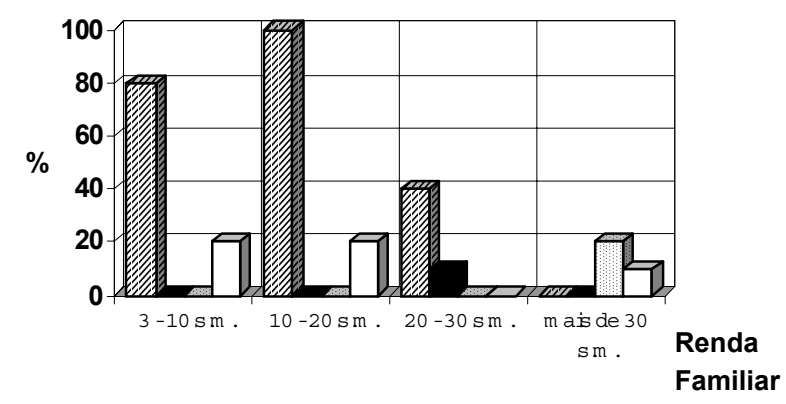

Figura 3 - Distribuição das mães de crianças e adolescentes com diabetes mellitus tipo 1 , segundo renda familiar, recursos e aparelhos existentes no domicílio para controlar o diabetes, Ribeirão Preto-SP, 1998.

Ao analisarmos a associação entre renda familiar, instrumentos e equipamentos existentes no domicílio, verificamos que todas as famílias com renda familiar entre 10 e 20 salários mínimos utilizam somente seringa, agulha, algodão e álcool para 0 controle domiciliar do diabetes. $\mathrm{Na}$ faixa salarial de 20 a 30 salários mínimos, $10 \%$ das famílias têm, além dos recursos mencionados, também o vitajet; $20,0 \%$, com renda superior a 30 salários mínimos possuem o glucometer e somente $20,0 \%$ com renda familiar de 3 de 10 salários mínimos e de 10 a 20 salários mínimos têm no domicílio as fitas reagentes para o controle do diabetes.

Os resultados indicam que há associação entre renda familiar, instrumentos e quipamentos para controle do diabetes. No entanto, acreditamos que a falta de informação sobre os mesmos, aliada aos seus custos elevados e à falta de reconhecimento dos pais sobre a importância do controle domiciliar para minorar as futuras complicações para a criança e o adolescente, possam estar interferindo na aquisição destes instrumentos e equipamentos e, conseqüentemente, na utilização para o controle domiciliar do diabetes.

Em relação à fonte de informação sobre o diabetes mellitus, quase metade das mães $(46,6 \%)$ obteve com o médico, livros e pessoas amigas. Observamos ainda que entre os membros da equipe de saúde, além do médico, somente 0 enfermeiro foi citado por 7 mães $(13,3 \%)$ como referência para informação. Um outro dado mostrou que as mães têm procurado outras mães com filhos diabéticos para obter informações.

Entendendo que 0 tratamento do diabetes mellitus em criança e adolescente envolve vários fatores, exigindo um acompanhamento seguro e contínuo por parte dos pais e da própria criança e adolescente para um bom controle metabólico, urge

\footnotetext{
* glucometer - aparelhos de fácil transporte e manuseio, que utilizam fitas reagentes para determinações da glicemia

** vitajet U 100 - injetor de insulina a jato, sem agulha, e por pressão de ar. Utiliza frasco de insulina comum, facilmente recarregável
} 
intensificarmos a implantação de programas com equipes multidisciplinares que ofereçam apoio a esta clientela.

Ao investigarmos a participação das mães nos grupos de educação em diabetes, verificamos que $19(63,3 \%)$ não estão inseridas em nenhum deles e 11 (36,7\%) disseram participar. Apresentamos a seguir os motivos pelos quais algumas mães não estão engajadas em grupos de orientações:

... é duro falar sobre isso ...

... eu não sei da existência de grupos ...

... porque é sempre a mesma coisa, para mim, isto não resolve ... ... não vou mais, fui algumas vezes e fiquei deprimida ...

Apesar de amplamente divulgada na literatura, a educação em diabetes continua sendo uma tarefa difícil, pois, a educação deve ser vista sob vários aspectos, ou seja, sob o ponto de vista da criança e do adolescente, da equipe multiprofissional e da família. Este conjunto de pessoas possui crenças, valores e mitos diferentes, que representam a sua própria visão de mundo, tornando complexa a ação educativa. Portanto, mudar comportamentos frente às práticas de saúde é uma tarefa árdua e contínua, que exige dos profissionais de saúde: tempo, recursos e disponibilidade para instrumentalizarse em termos de diabetes, de capacitação pedagógica, na busca de alternativas metodológicas que poderão sensibilizar as crianças e adolescentes para 0 auto-cuidado e apoiar a família no cuidado domiciliar.

Os dados indicam pequenos progressos nesta área, uma vez que as mães revelaram que não se sentem motivadas nem reconhecem a educação como um instrumento que ajude a criança e 0 adolescente a obterem um melhor controle metabólico.

\section{CONCLUSÕES E RECOMENDAÇÕES}

\author{
Esta investigação permitiu-nos concluir que:
}

- Para a metade dos casos estudados, a administração de insulina

\section{REFERÊNCIAS BIBLIOGRÁFICAS}

1. Diabetes Control and Complication Trial Research Group. The effect of intensive treatment of diabetes on the development and progression of long-term complications in insulin - dependent diabetes mellitus. $\mathrm{N}$ Engl J Med 1993; 329:977-86.

2. Brancati FL. The benefits of tight glycemic control in diabetes mellitus. Arch Intern Med 1995; 155(7):665-667.

3. Grossi SAA. Avaliação de dois esquemas de monitorização domiciliar em pacientes com diabetes mellitus do tipo 1. [dissertação]. São Paulo (SP): Escola de Enfermagem/USP; 1999. vem sendo realizada pela mãe e filho e que a maioria $(43,3 \%)$ aprendeu este procedimento com auxiliares de enfermagem ou "farmacêuticos".

- Os recursos utilizados no domicílio, para o controle do diabetes, são escassos; quando disponíveis, não são manuseados adequadamente.

- A comunicação das mães com a equipe de saúde mostrou-se limitada, sendo indicada como fonte de informação, em aproximadamente metade dos casos (46,6\%), os médicos, livros e pessoas amigas, sendo pequena a participação do enfermeiro.

Os dados encontrados nesta investigação merecem ser analisados pelos profissionais de saúde com vistas ao aprimoramento dos programas de assistência às crianças e adolescentes diabéticos. Neste sentido, delimitamos algumas recomendações, as quais apresentamos a seguir:

- Formação e capacitação de equipes multiprofissionais especializadas para atenderem às crianças e adolescentes diabéticos tipo 1, os quais exigirão esforços conjuntos entre instituições públicas e privadas.

- Fortalecimento e ampliação de grupos de educação em diabetes que motivem as mães a participar efetivamente deles, dando-Ihes oportunidade de elaboração dos problemas enfrentados com os filhos.

- Reconhecimento do enfermeiro, enquanto profissional da equipe de saúde, como responsável pelo acompanhamento domiciliar das famílias com crianças e adolescentes diabéticos, elemento que deve comprometer-se com a educação e treinamento dos pacientes e seus familiares, incluindo-se instrumentos e equipamentos utilizados no domicílio.

- Eqüidade no atendimento às famílias de crianças e adolescentes diabéticos tipo 1, fornecendo-lhes apoio para lidarem com a criança portadora de doença crônica.

4. Insulina: o factor econômico. Saúde do Mundo junho 1991; (Número Especial): 11.

5. Sabbeth B. Understanding the impact of chronic childhood illness on families. Pediatr Clin North Am 1984; 31(1):47-57.

6. Murphy H. A diabetes na infância. Nurs Rev Técnica Enfermagem 1995; 8(92):20-3.

7. Secretaria da Saúde (Ribeirão Preto). Programa de diabetes da Secretaria Municipal da Saúde. Ribeirão Preto; 1992.

8. Secretaria da Saúde (Ribeirão Preto). Proposta de plano de prevenção e controle do diabetes mellitus no município de Ribeirão Preto. Ribeirão Preto; 1993. 